\title{
Graph Theoretic Models and Tools for the Analysis of Dynamic Wireless Multihop Networks
}

\author{
Guoqiang Mao *† \\ ${ }^{*}$ School of Electrical and Information Engineering \\ The University of Sydney \\ ${ }^{\dagger}$ National ICT Australia Limited[1], Sydney, Australia \\ Email: guoqiang@ee.usyd.edu.au
}

\author{
Brian D.O. Anderson ${ }^{\ddagger \S}$ \\ $\ddagger$ Research School of Information Sciences and Engineering \\ Australian National University

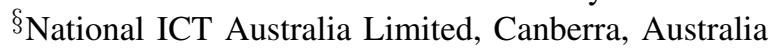 \\ Email: brian.anderson@nicta.com.au
}

\begin{abstract}
Wireless multihop networks are being increasingly used in military and civilian applications. Advanced applications of wireless multihop networks demand better understanding on their properties. Existing research on wireless multihop networks has largely focused on static networks, where the network topology is time-invariant; and there is comparatively a lack of understanding on the properties of dynamic networks with dynamically changing topology. In this paper, we use and extend a recently proposed graph theoretic model, i.e. evolving graphs, to capture the characteristics of such networks. We extend and develop the concepts of route matrix, connectivity matrix and probabilistic connectivity matrix as convenient tools to characterize and investigate the properties of evolving graphs and the associated dynamic networks. The properties of these matrices are established and their relevance to the properties of dynamic wireless multihop networks are introduced.
\end{abstract}

Index Terms-evolving graphs, connectivity, throughput

\section{INTRODUCTION}

Wireless multihop networks, in various forms e.g. wireless mesh networks, opportunistic networks, delay tolerant networks, mobile ad-hoc networks, vehicular ad-hoc networks and wireless sensor networks, are being increasingly used in military and civilian applications. Wireless multihop networks can be broadly classified into static networks, where the network topology is time-invariant, and dynamic networks with dynamically changing topology. Advanced applications of wireless multihop networks demand better understanding on the fundamental properties of these networks.

Despite significant research into the properties of static wireless multihop networks, there is much less understanding on the properties of dynamic multihop networks. "Most results found in the literature either deal with networks that are static or are hardly reproducible" [7]. This lack of understanding on the properties of dynamic multihop networks is attributable to a lack of a set of formal tools to carry out formal theoretical analysis [11].

In wireless multihop networks a change in topology can be caused by node mobility, by node/link failure or by nodes

\footnotetext{
${ }^{1}$ National ICT Australia is funded by the Australian Government Department of Communications, Information Technology and the Arts and the Australian Research Council through the Backing Australia Ability initiative and the ICT Centre of Excellence Program.
}

being switched on/off for energy saving purposes ${ }^{1}$. There are two major differences between a static network and a dynamic network:

- In dynamic networks, it is possible that two nodes may never be part of the same connected component but they are still able to communicate with each other within a finite time. This is illustrated in Fig. 1.

- In dynamic networks, while any one wireless link may be (or may be assumed to be) undirectional, the path connecting any two nodes must be regarded as directional, i.e. the fact that there is a path from node $v_{i}$ to node $v_{j}$ within a designated time period does not necessarily mean there is a path from $v_{j}$ to $v_{i}$ within the same period. For example, in Fig. 1 a message from $v_{6}$ can reach $v_{1}$ at $t_{2}$ but a message from $v_{1}$ can only reach $v_{6}$ at $t_{4}$.

Therefore many established concepts in static networks must be revisited for dynamic networks. For example, a static wireless multi-hop network is said to be connected if there is a path between any pair of nodes in the network. A static wireless multi-hop network is $k$-connected if there are $k$ mutually independent paths, i.e. paths not having any nodes in common except the starting and ending nodes, between any pair of nodes in the network. However a more meaningful definition of connectivity in dynamic networks is to say that a dynamic network is connected in time period $[0, T]$ if there is a path from any node to any other node within $[0, T]^{2}$. The above definition implies that the tradeoff between connectivity, mobility (or active period in networks with stationary nodes but nodes may be switched on/off) and delay must be properly considered in the analysis of dynamic networks. Indeed, it was shown in [8] that in a mobile network with no delay requirement, mobility of nodes can be exploited to significantly increase network capacity [8], [11]. Similarly it can also be expected that mobility can be exploited to improve connectivity. It remains an open problem to investigate the connectivity and other properties, e.g. end-toend delay, throughput, of dynamic multihop wireless networks.

\footnotetext{
${ }^{1}$ Topology change caused by temporal changes in wireless links is not included because this effect can usually be addressed by a modification of the models and analysis for static wireless multihop networks.

${ }^{2} \mathrm{~A}$ rigorous definition of connectivity in dynamic networks is given in Section III.
} 


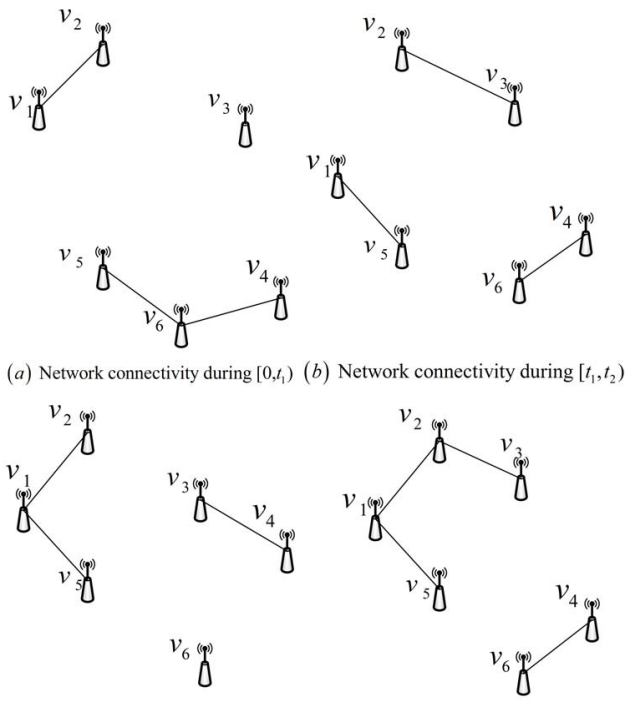

(c) Network connectivity during $\left[t_{2}, t_{3}\right)(d)$ Network connectivity during $\left[t_{3}, t_{4}\right)$

Figure 1. An illustration of connectivity in mobile ad-hoc networks with six nodes. A solid line represents a direct connection between two nodes. The network is disconnected at any time instant but there is a path from any node to any other node in the network. For example, nodes $v_{1}$ and $v_{6}$ are never part of the same connected component but a message from $v_{1}$ can still reach $v_{6}$ through the following path: $\left[0, t_{1}\right): v_{1} \rightarrow v_{2},\left[t_{1}, t_{2}\right): v_{2} \rightarrow v_{3}$, $\left[t_{2}, t_{3}\right): v_{3} \rightarrow v_{4},\left[t_{3}, t_{4}\right): v_{4} \rightarrow v_{6}$.

In this paper, we use and extend a recently proposed graph theoretic model, i.e. evolving graphs, to capture the characteristics, in particular the connectivity, of dynamic wireless multihop networks. We extend and develop a set of tools, i.e. route matrix, connectivity matrix and probabilistic connectivity matrix, to investigate the properties of dynamic multihop wireless networks. The properties of these matrices related to dynamic multihop networks are established. The rest of the paper is organized as follows. In Section II we briefly review related work in the area. In Section III we introduce and extend the concept of evolving graphs as a formal abstraction of dynamic networks. In Section IV we develop the route matrix, connectivity matrix and probabilistic connectivity matrix as convenient algebraic tools to investigate evolving graphs. The properties of these matrices are established. Finally conclusions and further work are given in Section V.

\section{RELATED WORK}

A graphic model of a wireless multihop network can be established by representing a node in the network uniquely by a vertex in the graph and by representing a wireless link uniquely by an edge in the graph, and vice versa. For simplicity, it is often assumed that the wireless link is symmetric and can be represented by an undirected edge [14], [11]. The obtained undirected graph $G(V, E)$ where $V$ is the vertex set and $E$ is the edge set is called the underlying graph of the wireless multihop network. A widely used model for static wireless multihop networks is random geometric graphs (RGG) [14]. In random geometric graphs, vertices are i.i.d. distributed in a given area following either a uniform distribution or Poisson distribution. An edge exists between two vertices iff their Euclidean distance is less than or equal to a given threshold, known as the transmission range. This communication model is known as the unit disk communication model.

In the late 1990s and early 2000s, Penrose [14], Gupta and Kumar [10], and Appel and Russo [1] investigated the connectivity of static wireless multihop networks using RGG and showed that in a network consisting of $n$ nodes identically, independently, randomly and uniformly distributed on a disk of unit area in $\Re^{2}$ and following the unit disk communication model, the critical transmission range $r_{c}(n)$ for the network to be connected is $\sqrt{\frac{\log n}{\pi n}}$ in the sense that the network with $r(n)=\sqrt{\frac{\log n+c(n)}{\pi n}}$ is asymptotically connected with probability one iff $c(n) \rightarrow+\infty$ as $n \rightarrow \infty$; the network is asymptotically disconnected with probability one iff $c(n) \rightarrow$ $-\infty$ as $n \rightarrow \infty$. Penrose further demonstrated [14] that in such a network, the critical transmission range $r_{k}(n)$ required for the network to be $k$-connected and the critical transmission range $\tau_{k}(n)$ required for the network to have a minimum node degree $k$ converge to the same value as $n \rightarrow \infty$, i.e. $\lim _{n \rightarrow \infty} \operatorname{Pr}\left\{r_{k}(n)=\tau_{k}(n)\right\}=1$. These fundamental results on network connectivity form the basis of the work by hundreds of researchers on designing energy efficient routing and scheduling algorithms, topology control and coverage control, localization, and radio resource management, see [12], [18] and references therein for example. There are many other results on the connectivity of static multihop networks. These results [14], [10], [1] on network connectivity however only apply to static networks, and it remains an open problem to investigate the connectivity of dynamic multihop wireless networks along similar lines.

The most related work to ours includes the use of adjacency matrix to study the connectivity of networks by Cvetkovic et al. [4] and Brooks et al. [3]. In [4], the adjacency matrix of a static network of $n$ nodes is defined to be an $n \times n$ square matrix $M$ whose $(i, j)$-th entry $m_{i j}=1$ if there is a direct connection between distinct nodes $i$ and $j$, otherwise $m_{i j}=0$. Further $m_{i i}$ is defined to be 0 , i.e. no self-loop is allowed. It is shown that the number of walks of length $z$ between nodes $i$ and $j$ (with $j=i$ permitted) is $m_{i j}^{z}$, where $m_{i j}^{z}$ is the $(i, j)$-th entry of $M^{z}$. A walk of length $z$ between nodes $i$ and $j$ is a sequence of $z+1$ nodes $i, v_{1}, v_{2}, \ldots, v_{z-1}, j$ which starts at $i$ and ends at $j$ and there is a direct connection between adjacent nodes. A path of length $z$ between nodes $i$ and $j$ is a walk of length $z$ between nodes $i$ and $j$ in which the vertices $i, v_{1}, v_{2}, \ldots, v_{z-1}$ are distinct, save that $i=j$ is permitted. If $i=j$, the path is called a cycle. In [3] Brooks et al. considered a probabilistic version of the adjacency matrix for multihop networks and define a probabilistic adjacency matrix $^{3}$ as an $n \times n$ square matrix $M$ whose $(i, j)$-th entry $m_{i j}$ represents the probability of having a direct connection

\footnotetext{
${ }^{3}$ It is called connectivity matrix in [3] but is more widely referred to as adjacency matrix in the literature. In this paper, we call it adjacency matrix to differentiate it from the connectivity matrix defined later in the paper.
} 


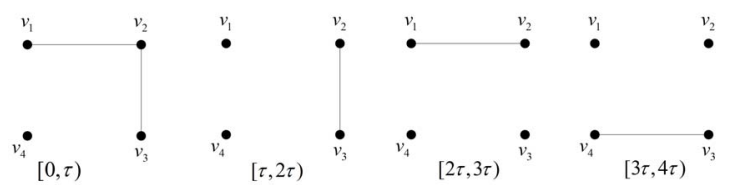

Figure 2. An illustration of the connectivity of a dynamic network in different time intervals. Each subfigure represents the connectivity of a fournode network in a corresponding time interval. A solid line represents a direct connection between two nodes.

between distinct nodes $i$ and $j$, and $m_{i i}=0$. They established that the probability that there exists at least one walk of length $z$ between nodes $i$ and $j$ is $m_{i j}^{z}$, where $m_{i j}^{z}$ is the $(i, j)$-th entry of $M \otimes M \otimes \cdots \otimes M$ ( $z$ times). $C \doteq A \otimes B$ iff $C_{i j}=$ $1-\prod_{l \neq i, j}\left(1-A_{i l} B_{l j}\right)$ where $A_{i j}, B_{i j}$ and $C_{i j}$ are the $(i, j)$-th entries of the $n \times n$ square matrix $A, B$ and $C$ respectively. We remark that the product defined this way has the associativity property, allowing the computation of a product of three or more matrices with an arbitrary sequence of single product operations.

The above adjacency matrix and probabilistic adjacency matrix, however, is not suitable for investigating dynamic networks. This is illustrated through the example dynamic network of four nodes shown in Fig. 2. Denote the adjacency matrix of the network during $[(i-1) \tau, i \tau)$ by $M_{i}$. If one were to follow the above definition of adjacency matrix, it would be concluded that there is no connection from $v_{1}$ to $v_{4}$ in the network within $[0,4 \tau)$ because the $(1,4)$-th entries in $M_{1}, M_{1} M_{2}, M_{1} M_{2} M_{3}$ and $M_{1} M_{2} M_{3} M_{4}$ are all zero. This is obviously incorrect because a message from $v_{1}$ can reach $v_{4}$ using the following journey ${ }^{4}:[0, \tau): v_{1} \rightarrow v_{3}$, $[\tau, 2 \tau): v_{2} \rightarrow v_{3},[3 \tau, 4 \tau): v_{3} \rightarrow v_{4}$. If the $1 \mathrm{~s}$ in $M_{i}, 1 \leq i \leq 4$ are replaced by values between 0 and 1 representing the probabilities that there are direct connections between the corresponding pairs of vertices, $M_{i}, 1 \leq i \leq 4$ becomes the probabilistic adjacency matrix of the network during $[(i-1) \tau, i \tau)$ as defined in [3]. It can be obtained that the probability that there exists a connection from $v_{1}$ to $v_{4}$ in the network within $[0,4 \tau)$ is zero because the $(1,4)$-th entries in $M_{1}, M_{1} \otimes M_{2}, M_{1} \otimes M_{2} \otimes M_{3}$ and $M_{1} \otimes M_{2} \otimes M_{3} \otimes M_{4}$ are all zero. Apparently this is not true. Therefore neither the adjacency matrix defined in [4] and the probabilistic adjacency matrix defined in [3] is suitable for investigating the connectivity of dynamic networks. The problem with the definition of Brooks et al. [3] is that it fails to capture the notion that in a wireless hop network, it is legitimate for a message to remain at a node over one or more time intervals.

Other related work includes [15] and [5]. In [15] Santi and Blough empirically investigated the connectivity of mobile adhoc networks for different combinations of number of nodes $n$ and transmission range $r$ using the random walk mobility model [9]. In [5] Díaz et al. analyzed the connectivity of a mobile ad-hoc network in which a total of $m$ nodes move randomly along the edges of a $n \times n$ grid.

\footnotetext{
${ }^{4}$ We will give a formal definition of the term journey in Section III.
}

\section{EVOLVING GRAPH THEORY}

A major challenge in the analysis of dynamic networks is choosing a proper graphic model that can both capture the dynamics of the network and be tractable for analytical studies. We use and extend a class of graphs, evolving graphs [2], [7], [17], as a formal abstraction of dynamic networks for our analysis. There has been research in the literature on the evaluation of computational complexity of algorithms in evolving graphs [17], [2] and on simulation based studies on the performance of routing algorithms in dynamic networks using evolving graphs [13].

An evolving graph $\mathcal{G}=\left(G, S_{G}\right)$ consists of a given graph $G(V, E)$, along with an ordered sequence of its subgraphs, $S_{G}=G_{1}\left(V_{1}, E_{1}\right), G_{2}\left(V_{2}, E_{2}\right), \ldots, G_{\tau}\left(V_{\tau}, E_{\tau}\right), \tau \in \mathbb{N}$. Let the vertex set and the edge set of $\mathcal{G}$ be $V_{\mathcal{G}}=\cup V_{i}$ and $E_{\mathcal{G}}=\cup E_{i}$ respectively. If we assign a physical meaning to subgraphs such that the subgraph $G_{i}\left(V_{i}, E_{i}\right)$ at a given index $i$ is the underlying graph of the network during the time interval $\left[t_{i-1}, t_{i}\right)$ and $t_{0}<t_{1}<\cdots<t_{\tau}$, the time domain is then incorporated into the model. Let $P_{i}$ be a path in $G_{i}$. Let $F\left(P_{i}\right)$ and $L\left(P_{i}\right)$ be the first vertex and the last vertex of $P_{i}$ respectively and $\left|P_{i}\right|$ be its length. We define a journey in $\mathcal{G}=\left(G, S_{\mathcal{G}}\right)$ from vertex $u$ to vertex $v$ of $V_{\mathcal{G}}$ as a sequence $J(u, v)=P_{\lambda 1}, P_{\lambda 2}, \ldots, P_{\lambda l}$, with $1 \leq \lambda 1<\lambda 2<\cdots<\lambda l \leq \tau$, such that (a) $P_{\lambda i}$ is a path in $G_{\lambda i}$ with $F\left(P_{\lambda 1}\right)=u, L\left(P_{\lambda l}\right)=v$; (b) for all $1 \leq i \leq l$, it holds that $F\left(P_{\lambda i}\right) \neq L\left(P_{\lambda i}\right)$ and (c) for all $i<\bar{l}$, it holds that $L\left(P_{\lambda i}\right)=F\left(P_{\lambda i+1}\right)$. The length of the journey is $\sum_{i=1}^{l}\left|P_{\lambda i}\right|$. The restriction $F\left(P_{\lambda i}\right) \neq L\left(P_{\lambda i}\right)$ has limited the journey to consist of non-cycle paths only. Nevertheless a vertex may still appear more than once inside a journey, viz a vertex might appear both in a path $P_{\lambda i} \in J(u, v)$ and in a path $P_{\lambda j} \in J(u, v)$, where $\lambda i \neq \lambda j$. The starting and the ending vertices of a journey may also be the same. We define an evolving path in $\mathcal{G}=\left(G, S_{\mathcal{G}}\right)$ from vertex $u$ to vertex $v$ of $V_{\mathcal{G}}$ as a journey $J(u, v)$ in which all vertices are distinct except that the ending vertex of the journey may be the same as the starting vertex. The term evolving path is intended to differentiate a path in an evolving graph from a path in one of the subgraphs $G_{\lambda i}$. A vertex $v$ is said to be $k$-hops away from another vertex $u$ if the length of the shortest journey (or equivalently the shortest evolving path) from vertex $u$ to vertex $v$ is $k$. The evolving graph $\mathcal{G}=\left(G, S_{\mathcal{G}}\right)$ is said to be a connected graph if there exists a journey (or equivalently an evolving path) from any vertex to any other vertex of $V_{\mathcal{G}}$.

\section{Connectivity Matrix And Probabilistic CONNECTIVITY MATRIX FOR DYNAMIC NETWORKS}

To facilitate later discussions, we call a dynamic network deterministic if all entries of its adjacency matrix, as defined in [4], during each time interval are either 1 or 0 , representing the fact that there are or there are no direct connections between the corresponding pairs of vertices respectively. We call a dynamic network probabilistic if during some time intervals only statistical information on the probabilities that there are direct connections between certain pairs of nodes is known. 


\section{A. Connectivity Matrix of Deterministic Dynamic Networks}

Let $0, \tau_{1}, \tau_{2}, \ldots, \tau_{k}, \ldots, 0<\tau_{1}<\tau_{2}<\cdots<\tau_{k} \cdots$ be the embedding points of the network such that network topology, defined by a collection of all direct connections between nodes in the network, only changes at these discrete time instants. We define the route matrix $H_{k}$ of a deterministic dynamic network of $n$ nodes during time interval $\left[\tau_{k-1}, \tau_{k}\right)$ whose underlying graph is $G_{k}$ as an $n \times n$ square matrix such that its $(i, j)$ th entry is the number of distinct paths in $G_{k}$ from vertex $i$ to another distinct vertex $j$. If there is no path from $i$ to $j$, $\left(H_{k}\right)_{i j}=0$ where $\left(H_{k}\right)_{i j}$ is the $(i, j)$-th entry of $H_{k}$. Further the diagonal entries of $H_{k}$ is 1 by definition. As will be shown later, this definition of the diagonal entries properly reflects the fact that in dynamic networks routes and connections are constantly changing, when a node either becomes isolated or cannot find a (better) route to the destination, it has to store the message until a (better) route is available. For convenience, we assume that $V_{1}=V_{2}=\cdots=V_{k}=\cdots$ such that the route matrices during different time intervals have the same dimension. If a node disappears and then reappears in the network, the situation can be readily accommodated by considering that node as an isolated node during some time intervals.

Based on the above definition of the route matrix, we can establish some properties of the route matrix and their relevance to the properties of the dynamic network.

Lemma 1: There are a total of $H_{i j}^{k}$ journeys from node $i$ to node $j$ in the evolving graph $\mathcal{G}=\left(G, S_{G}\right), S_{G}=$ $G_{1}\left(V_{1}, E_{1}\right), G_{2}\left(V_{2}, E_{2}\right), \ldots, G_{k}\left(V_{k}, E_{k}\right), \quad k \in \mathbb{N}$ within $\left[0, \tau_{k}\right)$, where $H_{i j}^{k}$ is the $(i, j)$-th entry of $H_{1} H_{2} \cdots H_{k}-I$.

Proof: We prove Lemma 1 by iteration. When $k=1$, the lemma is obviously correct. Note that the diagonal entries of $H_{1}-I$ is 0 , i.e. there is no journey from a node to itself within $\left[0, \tau_{1}\right)$. When $k=2$,

$$
H_{i i}^{2}=h_{i i}^{1} h_{i i}^{2}+\sum_{l \neq i} h_{i l}^{1} h_{l i}^{2}-1=\sum_{l \neq i} h_{i l}^{1} h_{l i}^{2}
$$

is the total number of journeys from node $i$ to itself within $\left[0, \tau_{1}\right) \cup\left[\tau_{1}, \tau_{2}\right.$ ) (each journey is made of two non-cycle path, one from $i$ to $l \neq i$ in $G_{1}$ and the other from $l$ to $i$ in $G_{2}$ ), where $h_{i j}^{p}$ is the $(i, j)$-th entry of $H_{p}$. For distinct $i$ and $j$,

$$
H_{i j}^{2}=\sum_{l} h_{i l}^{1} h_{l j}^{2}=h_{i j}^{1}+h_{i j}^{2}+\sum_{l \neq i, j} h_{i l}^{1} h_{l j}^{2} .
$$

The first term in the above equation is the number of journeys from node $i$ to node $j$ made of paths in $G_{1}$ only; the second term is the number of journeys from $i$ to $j$ made of paths in $G_{2}$ only; and the third term is the number of journeys from node $i$ to node $j$, where each journey is made of a non-cycle path in $G_{1}$ and a non-cycle path in $G_{2}$. Therefore the lemma is true for $k=2$.

Assume the lemma is correct for $k=T$, let us consider $k=T+1$ and $i \neq j$.

$$
H_{i j}^{T+1}=\sum_{l} H_{i l}^{T} h_{l j}^{T+1}=H_{i j}^{T}+H_{i i}^{T} h_{i j}^{T+1}+\sum_{l \neq i, j} H_{i l}^{T} h_{l j}^{T+1} .
$$

In the above equation, the first term is the number of journeys from $i$ to $j$ within $\left[0, \tau_{T}\right)$. The sum of the second and the third terms is the number of newly added journeys from $i$ to $j$ where each journey consists of a non-cycle path in $G_{T+1}$. The second term is the sum of $\left(H_{i i}^{T}-1\right) h_{i j}^{T+1}$, representing the number of journeys that (a) start at $i$ before $\tau_{T}$; (b) end at $i$ at $\tau_{T}$; and (c) reach $j$ at $\tau_{T+1}$, and $h_{i j}^{T+1}$, representing the number of journeys from $i$ to $j$ made of non-cycle paths in $G_{T+1}$ only; and the third term is the number of journeys that end at an intermediate node at $\tau_{T}$ and reach $j$ at $\tau_{T+1}$. The sum of the three terms gives the total number of journeys from node $i$ to node $j$ within $\left[0, \tau_{T+1}\right)$. It is trivial to show that $H_{i i}^{T+1}-1$ is the total number of journeys that start and end at $i$ within $\left[0, \tau_{T+1}\right)$.

Remark 1: Denote by $H_{k}^{\prime}$ the route matrix of $G_{k}$ except that the diagonal entries of $H_{k}^{\prime}$ are zeros, i.e. $H_{k}^{\prime}=H_{k}-I$. It can be shown that

$$
H_{1} H_{2} \cdots H_{k}-I=\sum_{i} H_{i}^{\prime}+\sum_{i<j} H_{i}^{\prime} H_{j}^{\prime}+\cdots+H_{1}^{\prime} H_{2}^{\prime} \cdots H_{k}^{\prime} .
$$

The $r$-th sum on the right-hand side of the above equation represents the number of journeys with exactly $r$ non-cycle paths in $r$ distinct subgraphs of $\mathcal{G}$ respectively. Therefore the above definition of the route matrix and Lemma 1 allows the computation of the total number of journeys ever occurred in the evolving graph $\mathcal{G}$.

Lemma 2: A dynamic network is connected in $\left[0, \tau_{k}\right)$ iff $H_{1} H_{2} \cdots H_{k}>J$ element wise, where $J$ is an $n \times n$ matrix with all entries equal to 1 .

The proof of Lemma 2 is omitted.

Lemma 3: $H_{i j}^{k}$ is a non-decreasing function of $k$.

This lemma is an easy consequence of Lemma 1 and the fact that $H^{k}$ is the sum of $I$ and a nonnegative matrix. An implication of Lemma 3 is that in dynamic networks the number of journeys from any node to any other node will only increase over time.

We define the connectivity matrix $Q_{k}$ of a deterministic dynamic network of $n$ nodes whose underlying graph is $G_{k}$ during time interval $\left[\tau_{k-1}, \tau_{k}\right)$ as an $n \times n$ square matrix such that its $(i, j)$-th entry is 1 if there exists a path in $G_{k}$ from vertex $i$ to another distinct vertex $j$. If there is no path from $i$ to $j,\left(Q_{k}\right)_{i j}=0$. We also set $\left(Q_{k}\right)_{i i} \doteq 1$. Alternatively $Q_{k}$ can be considered as the sign matrix of $H_{k}$. Using the properties of non-negative matrices [16] and the above lemmas, we can establish the following properties of the connectivity matrix.

Lemma 4: A dynamic network is connected in $\left[0, \tau_{k}\right)$ iff $Q_{1} Q_{2} \cdots Q_{k} \geq J$ element wise. There is a journey from vertex $i$ to $j$ iff $\left(Q_{1} Q_{2} \cdots Q_{k}\right)_{i j} \geq 1$.

It can also be established that $\left(Q_{1} Q_{2} \cdots Q_{k}\right)_{i j}$ is a nondecreasing function of $k$. This, together with Lemma 4, suggests for a mobile network in which mobile nodes travel according to a random mobility model, e.g. random walk model [9], the network will become connected eventually. The interesting question is thus the rate at which the network becomes connected or equivalently the rate at which all entries 
of $Q_{1} Q_{2} \cdots Q_{k}$ (or equivalently $H_{1} H_{2} \cdots H_{k}$ ) become greater than or equal to 1 .

The connectivity matrix and the route matrix defined earlier, although are convenient tools to investigate connectivity, do not capture the fact that in a communications network a message can only travel a limited distance within a limited amount of time. In view of this, we extend the earlier definitions to account for message propagation delays. We call a path $P_{i}$ in $G_{i}$ feasible if a message of a unit length transmitted by the starting point of $P_{i}$ at $\tau_{i-1}$ can reach the end point of $P_{i}$ before $\tau_{i}$ in ideal conditions, i.e. no traffic congestion. We call a journey feasible if it is made entirely of feasible paths. We can also define the concept of feasible connectivity matrix and feasible route matrix analogously. Obviously the above lemmas on the properties of connectivity matrix and route matrix still hold if the relevant terms are replaced by their "feasible" counterparts. By a direct application of Lemma 1, we obtain the following Lemma that relates the feasible connectivity matrix to end-to-end throughput (or latency).

Lemma 5: Assuming that node $i$ starts to transmit a message of unit length to another distinct node $j$ at time 0 , there are $M_{i j}^{k}$ feasible journeys from $i$ to $j$ that take less than or equal to $\tau_{k}$ time for the unit message to reach $j$, where $M_{i j}^{k}$ is the $(i, j)$-th entry of $M_{1} M_{2} \cdots M_{k} . M_{k}$ is the feasible route matrix of graph $G_{k}$ during $\left[\tau_{k-1}, \tau_{k}\right)$.

Lemma 5 can be used to derive an upper bound on the endto-end throughput in a dynamic network. Further let $\tau$ be the time required to transmit a message of unit length from a node to its immediate neighbors. We choose the embedding points of the network $0, \tau_{1}, \tau_{2}, \ldots, \tau_{k}, \ldots$ to be $\tau_{k}=k \tau$ and assume that a node can only start transmitting a message at $\tau_{k}$ which accords with a synchronized network. If a direct connection between two nodes is broken during the interval $[(i-1) \tau, i \tau)$, it is considered that there is no direct connection between the two nodes during $[(i-1) \tau, i \tau)$. Under the above conditions, a feasible connectivity matrix becomes the sum of the corresponding adjacency matrix and $I$. We can obtain the following lemma:

Lemma 6: Assuming that node $i$ starts to transmit a unit message to another distinct node $j$ at time 0 , the least amount of time for the message to arrive at node $j$ is $k \tau$ iff $M_{i j}^{k-1}=0$ and $M_{i j}^{k} \geq 1$. The number of messages that can be transmitted from $i$ to $j$ within $[0, k \tau)$ is less than or equal to $M_{i j}^{k}$.

Lemmas 5 and 6 give an easy way to study the foremost journeys [17], [2] in an evolving graph. A slight modification of the two lemmas can be used to investigate the shortest journeys and the fastest journeys [17], [2] in an evolving graph algebraically.

\section{B. Probabilistic Connectivity Matrix of Probabilistic Dynamic Networks}

Let $0, \tau_{1}, \tau_{2}, \ldots, \tau_{k}, \ldots, 0<\tau_{1}<\tau_{2}<\cdots<\tau_{k} \cdots$ be the embedding points of the network such that network topology only changes at these discrete time points. We define the probabilistic connectivity matrix $Q_{k}$ of a probabilistic dynamic network of $n$ nodes during time interval $\left[\tau_{k-1}, \tau_{k}\right)$, whose underlying graph is $G_{k}$, as a $n \times n$ square matrix such that its $(i, j)$-th entry is the probability that there is a path in $G_{k}$ from vertex $i$ to another distinct vertex $j$ and in addition for all $i,\left(Q_{k}\right)_{i i} \doteq 1$. We further assume that

1) the events whose probabilities are the entries in the same probabilistic connectivity matrix are independent;

2) the events whose probabilities are the entries in probabilistic connectivity matrices during different time intervals are independent.

An example in which these assumptions hold is wireless sensor networks with uncoordinated power saving mechanisms [6]. We can establish the following properties of probabilistic connectivity matrix.

Lemma 7: The probability that there exists at least one journey from node $i$ to another distinct node $j$ in the evolving graph $\mathcal{G}=\left(G, S_{G}\right), S_{G}=$ $G_{1}\left(V_{1}, E_{1}\right), G_{2}\left(V_{2}, E_{2}\right), \ldots, G_{k}\left(V_{k}, E_{k}\right), k \in \mathbb{N}$ is $Q_{i j}^{k}$, where $Q_{i j}^{k}$ is the $(i, j)$-th entry of $Q_{1} \otimes Q_{2} \cdots \otimes Q_{k} . C=A \otimes B$ means $C_{i j}=1-\prod_{l}\left(1-A_{i l} B_{l j}\right)$, and $A_{i j}, B_{i j}$ and $C_{i j}$ are the $(i, j)$-the entries of $n \times n$ square matrices $A, B$ and $C$ respectively.

Proof: We prove this lemma by iteration and omit the proof that the defined product operation is associative. The lemma is obviously correct for $k=1$ and the diagonal elements $Q_{i i}^{1}=1,1 \leq i \leq n$.

Assuming the lemma is correct for $k=T$, let us consider $k=T+1$. First, it can be shown that the diagonal elements of $Q_{1} \otimes Q_{2} \cdots \otimes Q_{T+1}$ are always 1 . Let us now consider $Q_{i j}^{T+1}$ where $i \neq j$,

$$
Q_{i j}^{T+1}=1-\left(1-q_{i j}^{T+1}\right)\left(1-Q_{i j}^{T}\right) \prod_{l \neq i, j}\left(1-Q_{i l}^{T} q_{l j}^{T+1}\right)
$$

In the above equation, $q_{i j}^{T+1}$ is the $(i, j)$-th entry of $Q_{T+1}$ and represents the probability of having a journey consisting of a path from $i$ to $j$ in $G_{T+1}$ only. $Q_{i j}^{T}$ is the probability of having a journey from $i$ to $j$ in the evolving graph $\mathcal{G}=\left(G, S_{G}\right), S_{G}=G_{1}, G_{2}, \ldots, G_{T}$. The existence of such a journey necessarily means the existence of a journey in $\mathcal{G}=\left(G, S_{G}\right), S_{G}=G_{1}, G_{2}, \ldots, G_{T+1}$. The term $Q_{i l}^{T} q_{l j}^{T+1}$, $l \neq i, j$ is the probability of having a journey in $\mathcal{G}=\left(G, S_{G}\right)$, $S_{G}=G_{1}, G_{2}, \ldots, G_{T+1}$ that ends at an intermediate node $l$ at $\tau_{T}$ and reaches $j$ at $\tau_{T+1}$. Using the assumption on the independence of events represented by entries in the probabilistic connectivity matrix, we can obtain that the above three events are independent. Therefore $\prod_{l}\left(1-Q_{i l}^{T} q_{l j}^{T+1}\right)$ is the probability that none of the above three events occurs. Finally $1-\prod_{l}\left(1-Q_{i l}^{T} q_{l j}^{T+1}\right)$ gives the probability that at least one of the above three events occurs, which is the probability of having a journey from $i$ to $j$ in the evolving graph $\mathcal{G}=\left(G, S_{G}\right), S_{G}=G_{1}, G_{2}, \ldots, G_{T+1}$.

Lemma 8: $Q_{i j}^{k}$ is a non-decreasing function of $k$.

This lemma can be proved using the definition of $Q_{i j}^{k}$. Suppose that the probabilistic connectivity matrix is the same on every interval, so that the network simply resets certain connections, with the same probabilities, at each clock time. Then this 
lemma can be used to show that in the limit as $k$ tends to infinity, either $Q_{i j}^{k}$ tends to 1 , or it is zero for all $k$, i.e. there is never a connection from node $i$ to node $j$.

Lemma 9: A network of $n$ nodes is connected with probability 1 in $\left[0, \tau_{k}\right)$ iff $Q_{i j}^{k}=1$ for all pairs of $i$ and $j$.

This lemma is an easy consequence of Lemma 7.

Similarly by defining the concept of feasible probabilistic connectivity matrix, we can use the probabilistic connectivity matrix to investigate end-to-end delay. We define the feasible probabilistic connectivity matrix $Q_{k}$ of a probabilistic dynamic network of $n$ nodes during time interval $\left[\tau_{k-1}, \tau_{k}\right)$ as an $n \times n$ square matrix such that its $(i, j)$-th entry is the probability that there is a feasible path in $G_{k}$ from vertex $i$ to another distinct vertex $j$ and $\left(Q_{k}\right)_{i j} \doteq 1$. The above lemmas on the properties of probabilistic connectivity matrix still hold if the relevant terms are replaced by their "feasible" counterparts.

Further let $\tau$ be the time required to transmit a message of unit length from a node to its immediate neighbors. We choose the embedding points of the network $0, \tau_{1}, \tau_{2}, \ldots, \tau_{k}, \ldots$ to be $\tau_{k}=k \tau$ and assume a node can only start transmitting a message at $\tau_{k}$. If a direct connection is between two nodes is broken during the interval $[(i-1) \tau, i \tau)$, it is considered that there is no connection between the two nodes during $[(i-1) \tau, i \tau)$. Under the above conditions, a feasible probabilistic connectivity matrix becomes the sum of the corresponding probabilistic adjacency matrix, as defined in [3], and $I$. We can establish the following lemma:

Lemma 10: Assuming that node $i$ starts to transmit a unit message to a distinct node $j$ at time 0 , the probability that the message can reach $j$ in less than $\tau_{k}$ time is $Q_{i j}^{k}$, where $Q_{i j}^{k}$ is the $(i, j)$-th entry of $Q_{1} \otimes Q_{2} \cdots \otimes Q_{k}$ and the mean end-to-end delay from $i$ to $j$ is given by:

$$
\sum_{k=1}^{\infty}\left(1-Q_{i j}^{k-1}\right) Q_{i j}^{k} k \tau
$$

This lemma can be obtained from Lemma 7. It can be used to study the mean end-to-end delay and the delay distribution.

\section{CONCLUSION AND FURTHER WORK}

Wireless multihop networks is a fascinating area that has attracted growing interest. In this paper, we used and extended a recently proposed graph theoretic model, i.e. evolving graphs, as a formal abstraction of dynamic wireless multihop networks. We extended and developed a set of tools, i.e. route matrix, connectivity matrix and probabilistic connectivity matrix, to investigate the properties of dynamic multihop wireless networks. The properties of these matrices were investigated in relation to the properties of the corresponding dynamic multihop networks. We expect them to provide a convenient tool to formally investigate the properties of dynamic multihop networks.

An implicit hypothesis used in the paper is that a wireless node is able to store a message when it becomes isolated or cannot find better route to the destination. This has been the case in most wireless multihop networks, especially in an age when memory unit becomes increasing cheap and smallsized. However the tools developed in this paper can be easily modified to account for the situation that a node loses its message because of, e.g. limited battery power or buffer size.

Future work in the area will be carried out along two directions. First, we shall apply the model and tools to investigate the properties of dynamic networks, in particular the asymptotic properties of dynamic networks either when the number of nodes is large or when the time span of the associated evolving graph is long. Second, we shall further develop the tools to make them suitable for a wider category of dynamic multihop networks. For examples, the two independence assumptions in Section IV-B is a bit restrictive. In a mobile multihop network, the probabilistic connectivity matrices (adjacency matrices) during adjacent time intervals can be highly correlated. We expect to develop the tools to properly consider the time correlation among matrices.

\section{REFERENCES}

[1] M.J.B. Appel and R.P. Russo. The connectivity of a graph on uniform points on [0,1]d. Statistics and Probability Letters, 60(4):351-357, 2002.

[2] S. Bhadra and A. Ferreira. Complexity of connected components in evolving graphs and the computation of multicast trees in dynamic networks. In Lecture Notes in Computer Science, volume 2865, pages 259-270. Springer Berlin, 2003.

[3] R. R. Brooks, B. Pillai, S. Racunas, and S. A. Rai S. Rai. Mobile network analysis using probabilistic connectivity matrices. IEEE Transactions on Systems, Man, and Cybernetics, Part C: Applications and Reviews, 37(4):694-702, 2007.

[4] D. M. Cvetković, M. Doob, and H. Sachs. Spectra of Graphs. Johann Ambrosius Barth Verlag, 3rd edition, 1995.

[5] J. Diaz, D. Mitsche, and X. Perez. On the connectivity of dynamic random geometric graphs. 2007.

[6] O. Dousse, P. Mannersalo, and O. Thiran. Latency of wireless sensor networks with uncoordinated power savings mechanisms. In MOBIHOC, 2004.

[7] A. Ferreira. Building a reference combinatorial model for manets. IEEE Network, 18(5):24-29, 2004.

[8] M. Grossglauser and D. N. C. Tse. Mobility increases the capacity of ad hoc wireless networks. IEEE/ACM Transactions on Networking, 10(4):477-486, 2002.

[9] R. A. Guerin. Channel occupancy time distribution in a cellular radio system. IEEE Transactions on Vehicular Technology, 36(3):89-99, 1987.

[10] P. Gupta and P. R. Kumar. Critical power for asymptotic connectivity in wireless networks. In Stochastic Analysis, Control, Optimization and Applications, pages 547-566. Boston, MA: Birkhauser, 1998.

[11] P. Gupta and P.R. Kumar. The capacity of wireless networks. IEEE Transactions on Information Theory, 46(2):388-404, 2000.

[12] R. Hekmat. Ad-hoc Networks: Fundamental Properties and Network Topologies. Springer, 2006.

[13] J. Monteiro, A. Goldman, and A. Ferreira. Performance evaluation of dynamic networks using an evolving graph combinatorial model. In IEEE International Conference on Wireless and Mobile Computing, Networking and Communications, pages 173-180, 2006.

[14] M. D. Penrose. Random Geometric Graphs. Oxford Studies in Probability. Oxford University Press, USA, 2003.

[15] P. Santi and D.M. Blough. An evaluation of connectivity in mobile wireless ad hoc networks. In Proc. Int. Conference on Dependable Systems and Networks, pages $89-98,2002$.

[16] E. Seneta. Non-negative Matrices and Markov Chains. Springer Series in Statistics. Springer, 1973.

[17] B. Bui Xuan, A. Ferreira, and A. Jarry. Computing shortest, fastest, and foremost journeys in dynamic networks. International Journal of Foundations of Computer Science, 14(2):267-285, 2003.

[18] F. Xue and P. R. Kumar. Scaling Laws for Ad Hoc Wireless Networks: An Information Theoretic Approach, volume 1 of Foundations and Trends in Networking. Now Publishers Inc, 2006. 